Opinion

\title{
Spatial epidemiology in veterinary disease surveillance
}

\section{Introduction}

Geographical Information System (GIS) is a computer-based method to analyze and displaying digital geo-referenced data sets. Spatial epidemiology mainly focuses on the spatial distribution of a disease to identify the susceptible populations and possible etiological factors. The first requirement of epidemiological survey is to estimate disease incidence in order to concentrate the disease investigation establish the risk factors, to take preventive measures and to forecast the diseases. The aim of this paper is therefore to explore the usability of spatial epidemiology designed to improve the knowledge of veterinarians to control the diseases.

\section{Usage of spatial epidemiology}

a. Spatial epidemiology will display the disease distribution by spatial analysis along with the associated factors

b. Besides knowing the epidemic of disease location, it also helps in understanding the mode and pattern of transmission of an infectious disease.

c. Spatial maps can either be constructed according to shape of a district, state or country (called as geographic base maps) or according to size of population concerned (called as demographic/is demographic base maps).

d. The morbidity and mortality are presented in relation to the size of population in demographic type

e. Information on morbidity, mortality and population are greatly important for the maps designing.

f. Map will detect the possible causes of diseases of unknown etiology.

g. Maps will be qualitative or quantitative.

h. In qualitative type, the location of present/absence of disease is indicated. However, no information can be obtained regarding the amount of disease.

i. In quantitative type, the amount of a disease in a particular area is displayed.

\section{Several types of geographic base maps}

\section{Point (dot or location) map}

a. Show the location of a disease in a political map.

b. Locate by dot or circle, area can also be named.

\section{Distribution map}

a. Shows the area in which the disease occurs.

b. Example: Area endemic to disease, area sporadic to disease and area free from the disease are shown under different markings or shade.
Volume 9 Issue 5 - 2020

\author{
S Krishna Kumar,' KM Palanivel ${ }^{2}$ \\ 'Assistant Professor, Sheep Breeding Research Station, \\ Sandynallah, The Nilgiris, India \\ ${ }^{2}$ The Professor and Head, Department of Veterinary Preventive \\ Medicine, Veterinary College and Research Institution, India
}

Correspondence: S Krishna Kumar, Assistant Professor, Sheep Breeding Research Station, Sandynallah, The Nilgiris, Tamilnadu, India, Email subbiahkrishnakumar4@gmail.com

Received: February 20, 2020 | Published: October 30, 2020

\section{Choloroplethic map}

a. Display of quantitative information.

b. Example: Areas with $<15$ prevalence, $15 \%-30 \%$ prevalence, $30 \%-50 \%$ prevalence and $>50 \%$ prevalence of bovine viral diarrhea in a particular district (political map) are shown in different shades/markings.

c. Mapping according to administrative region is not a true boundary for incidence.

\section{Isoplethic map}

a. True boundary of disease is depicted in a geographic map, not region-wise as in a political map.

\section{Point (dot or location) map}

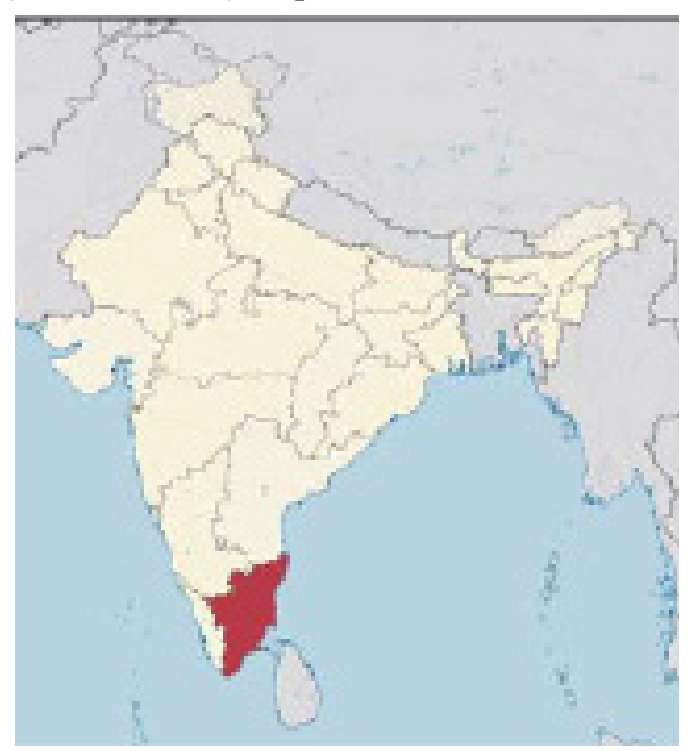




\section{Distribution map}

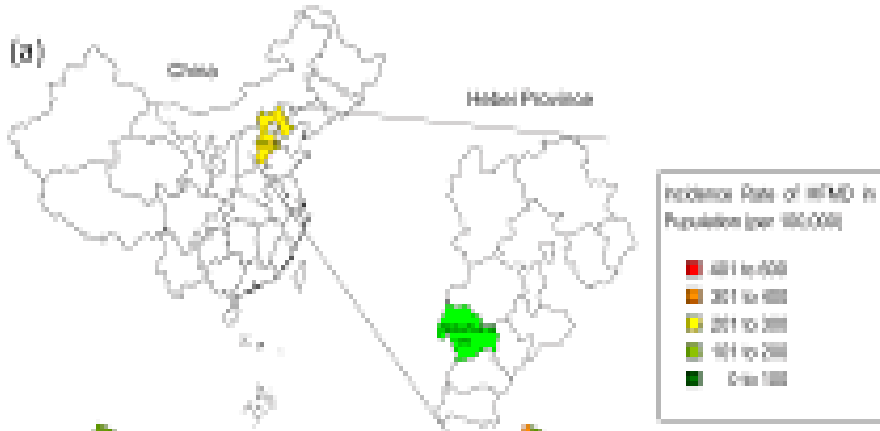

bi

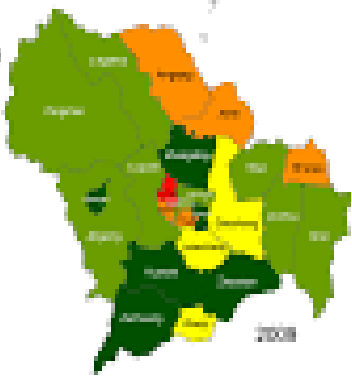

Id.

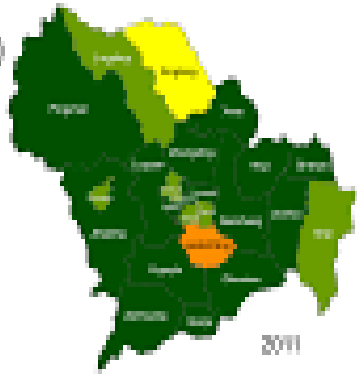

삼

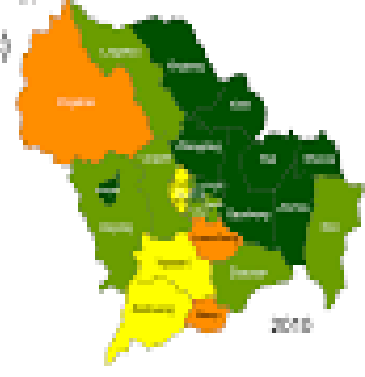

(b)

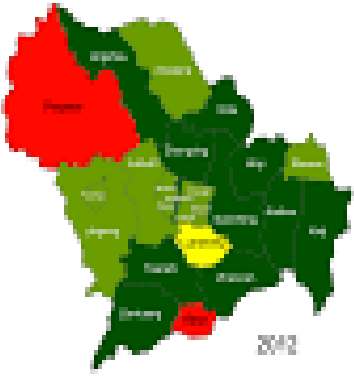

\section{Choloroplethic map}



\section{Isoplethic map}

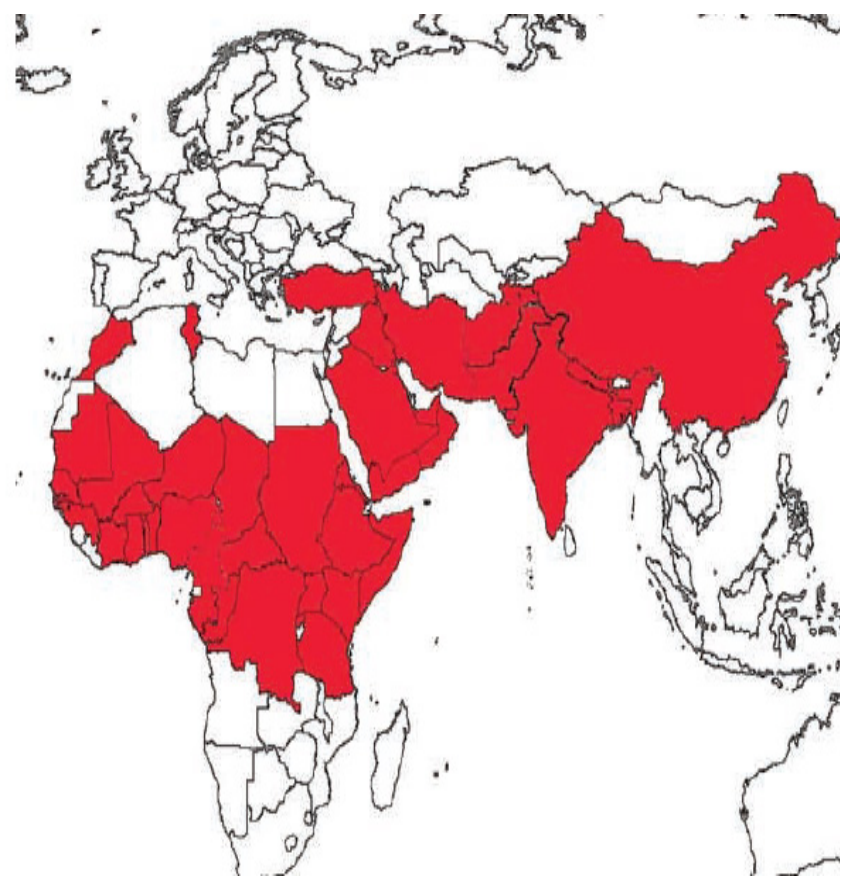

\section{Geographical information system}

Geographical Information System (GIS) is a computer based analyzing for displaying digital data with spatial sets. Spatial epidemiology mainly deals with the spatial/location epidemic of disease to identify the susceptible populations and epidemiological risk factors. Epidemiologic surveys will mainly calculate the disease incidence to facilitate the disease investigation and to frame preventive measures.

GIS displays the spatial data as subject matter layers which will be display one by one. These are stored in a spatial linked database. Each data will be stored, analyzed and retrieved as and when needed.

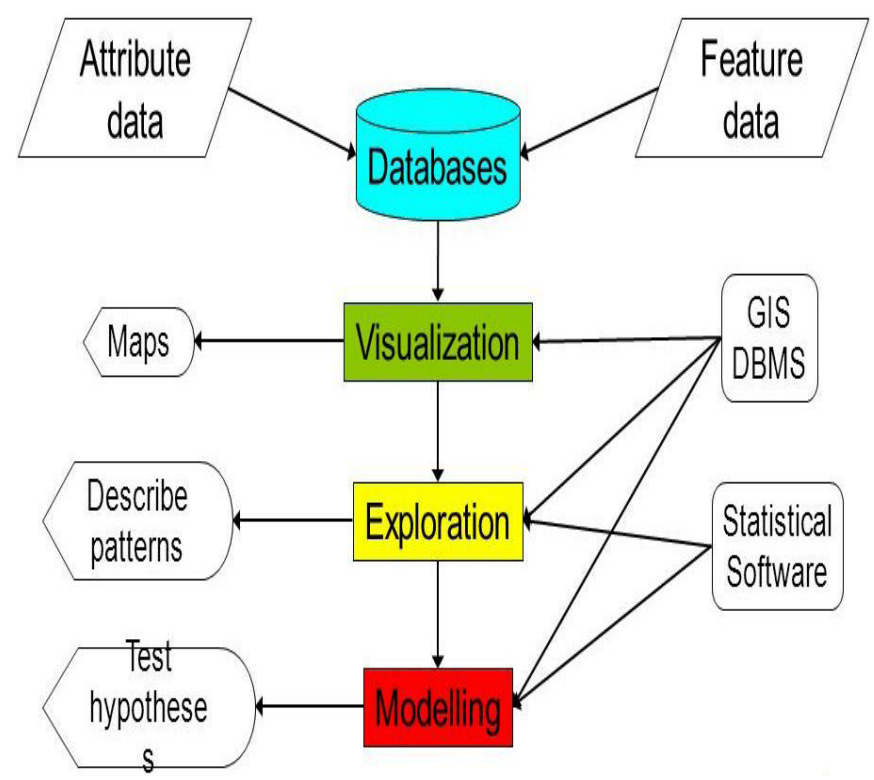




\section{GIS Data}

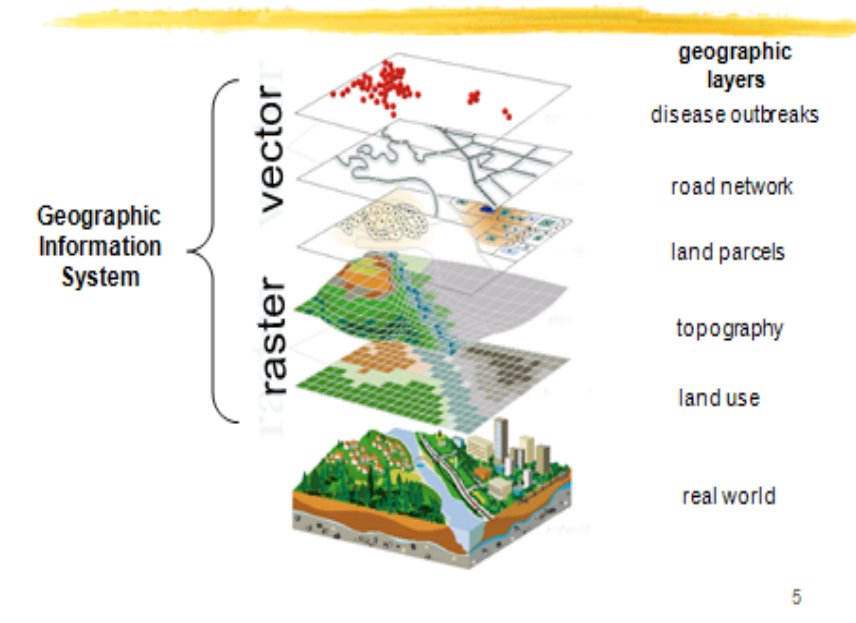

GIS application in the veterinary epidemiology

\section{a. Monitoring, recording and forecasting of disease}

GIS will be used to design maps of livestock disease incidence, mortality, morbidity pattern in a place/location. The information about the diseases will be enlightened with maps. Density of the population vs. diseases will be easily compared. This will give the ration ship data between disease and density risk factors.

\section{b. Epidemic emergency system}

In an epidemic situation, GIS can provide an detailed information about the location, other epidemiological risk factors etc., To control the ongoing diseases buffering the zone for vaccination is highly essential and it can be done by geo spatial study. Further, geo spatial maps will provide possible risk entry points of the diseases.

\section{c. Analysis of clustering of diseases in a place}

GIS will give time line data for a particular disease with relation to grouping.

\section{d. Modeling of spreading of disease}

Modelings are computer based software's where GIS will be utilized. Such models will include farm size, type of farm, density of animals, epidemic areas, type of soil with sloppiness and plantation pattern .These factors will be considered as epidemiological risk factors for disease epidemic.

\section{e. Planning of epidemic control programs}

The epidemiological tools like case control study and cohort study will through light on epidemic areas and its relationship for the development disease. Neighborhood analysis may be used to spot all adjoining farms to an affected farm. The spatial data will give idea about the disease spread and methods to control the disease.

\section{Conclusion}

Spatial epidemiology/GIS provide significant method or data to investigate/forecast the disease. Disease epidemic pattern, spreading methods, risk factors analysis will be improved with GIS. The epidemic of the disease will be studied well with spatial data which enable to control the disease.

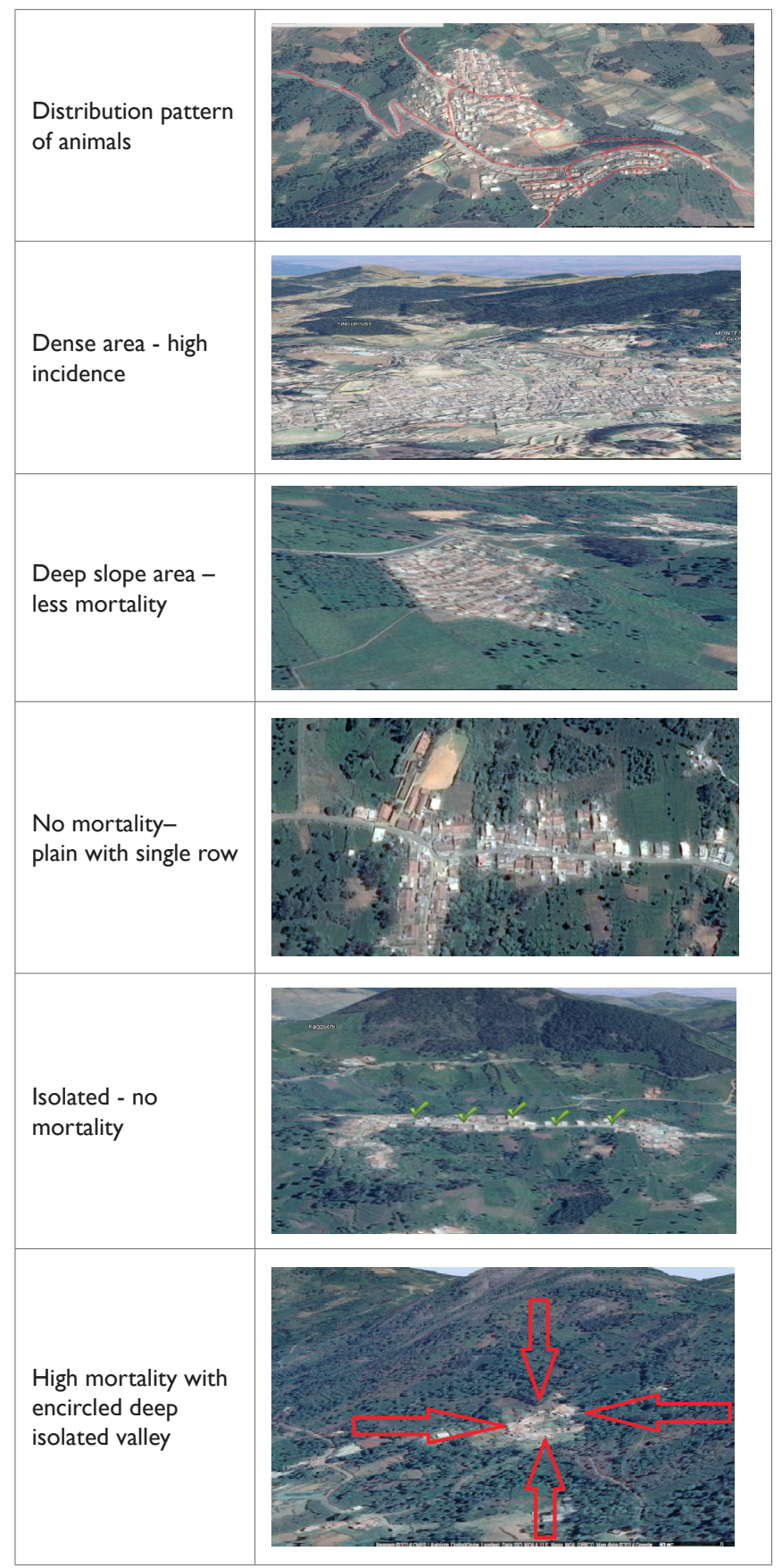

Acknowledgments

None.

\section{Conflicts of interest}

Author declares that there are no conflicts of interest. 